mirrors. The possibility had to be faced that the mirrors might suffer some distortion from the sun's heat. Apart from this, two of the mirrors did to some extent spoil the sharpness of the stellar images.

Three series of photographs were taken. Prof. Eddington and Mr. Cottingham at Principe had very cloudy weather, but obtained photographs showing a few stars. The check field obtained on two nights shortly after the eclipse gave images similar to those on the plates taken during the eclipse. The temperature during the eclipse was $77^{\circ} \mathrm{F}$., and $76.5^{\circ} \mathrm{F}$. when the check fields were taken. There was no reason to suppose any change of scale, and on this assumption the plates when measured gave for the deflection at the sun's $\operatorname{limb} \mathbf{r} \cdot 6 \mathrm{x}^{\prime \prime} \pm 0.30^{\prime \prime}$.

Dr. Crommelin and Mr. Davidson in Brazil were favoured with ideal weather conditions. They found, however, that the images on the eclipse plate differed from those taken the previous night, and from those taken on the same field of stars two months later. This is attributed to the effect of the sun's heat in distorting the colostat mirror. If it is assumed that the scale has changed, then the Einstein deflection from the series of plates is $0.90^{\prime \prime}$; if it is assumed that no real change of focus occurred, but merely a blurring of the images, the result is $\mathrm{x} \cdot 5^{6 / l}$; little weight is, however, attached to this series of photographs.

With a smaller lens of 4 -in. aperture and I9-ft. focus the same observers were extremely successful. The images taken during' the eclipse are in sharp focus and exactly similar to those on the comparison field. The result of measurement gave $1.98^{\prime \prime} \pm 0.12$ " for the deflection at the limb; seven stars were photographed, and the individual displacements, both in right ascension and in declination, were in good accordance with the law $r \cdot 75^{\prime \prime}(a / r)$. It has been pointed out by Prof. H. N. Russell that the photographs show a difference of scale of one part in 12,000 in the horizontal and vertical directions of the cœlostat mirror, and that if allowance be made for this the results for individual stars will be in still closer accordance.

The result of the eclipse, particularly of the second series of photographs at Sobral, is a close verification of Einstein's predicted displacements $1 \cdot 75^{\prime \prime}(a / r)$ radial from the sun's centre.

We proceed to consider the objections raised against this result. It has been suggested that allowance was not made correctly for the ordinary terrestrial refraction. The method adopted was that usually employed in astronomical photography, the second order terms being omitted, as they in no case amount to more than 0.02 ". The possibility of distortion of the film of the photographs, owing to the presence of the corona, has also been suggested. Examination of the plate in Phil. Trans., vol. ccxx., will show how different the conditions are from those cases in which distortion of the film has been observed. The possible displacements on the eclipse photographs could not amount to $0.05^{\prime \prime}$ for any of the stars, and would be in the wrong direction.

Prof. Anderson made the interesting suggestion that a possible error might arise from the fall of temperature in the air as the moon's shadow moves over the place of observation, thus causing an exceptional refraction. It has been shown by Prof. Eddington and Sir Arthur Schuster (NATuRE, vol. civ., pp. 372,468 ) that this effect would in all cases be very much smaller than the quantities in question. Further, it happens that at Sobral, owing to cloud in the early morning, which checked the normal daily rise of temperature, the fall during the eclipse was not more than ${ }_{2}^{\circ}$ or $3^{\circ} \mathrm{F}$.

The possibility that the observed displacements may be due to refraction by gaseous matter surrounding the sun has received a good deal of consideration. If such an atmosphere is controlled entirely by the sun's gravitation, an impossibly high density is required. Prof. Newall has investigated the consequences of assuming that gravitation is partly balanced near the sun by electrical forces and radiation pressure assumed to vary as the inverse square of the distance from the sun's centre. To obtain agreement with the eclipse observations, he finds it necessary that 0.999 of the weight of the gas should be thus balanced. The difficulties of such a supposition have been pointed out by Prof. Lindemann $(\mathrm{Ob}$ servatory, April, 1920). Further, Dr. Crommelin has directed attention to the fact that several comets have approached nearer the sun than paths of the rays of some of the stars photographed at the eclipse, yet their motion has not been retarded, or their substance entirely vaporised, although they were for two hours at this small distance from the sun and moving with a velocity of 300 kilometres a second.

\title{
Relativity and the Motion of Mercury's Perihelion.
}

\author{
By Dr. A. C. D. Crommelin.
}

THE effect of Einstein's law in changing the position of Mercury's perihelion is sometimes looked on as something mysterious and recondite; but in reality anything that disturbs the law of inverse squares in the smallest degree is bound to produce such a shift; and as in all the No. 2677 , voL. IO6] systems known to us such disturbing factors exist, the line of apses is invariably in motion.

It is easy to show that when the central force falls off more rapidly than the ratio of inverse squares, the apse-line advances; for we may consider the inverse square law as holding, with the 
addition of a small superposed force, outward in the outer half of the orbit, and inward in the inner half. These additional forces clearly cause the outward or inward motion to persist a little longer than it would otherwise do, thus delaying the attainment of the apses. It is proved in Tait and Steele's "Dynamics of a Particle," p. I27, that if the force vary as $r^{n}$, the apsidal angle in a nearly circular orbit is $\pi(3+n)^{-\frac{3}{3}}$. Put $n=-2(1+d)$, where $d$ is very small, then the apsidal angle is $\pi(\mathrm{I}-2 d)^{-\frac{1}{2}}=\pi(\mathrm{I}+d)$. Or, in a complete revolution, the apse advances $2 \pi d$, which is a constant for all orbits.

This was the hypothesis advanced by Prof. Asaph Hall to explain the motion of Mercury's apses. The shift in the case of Mercury is O.I" per revolution. Applying this to the moon, it would give an apsidal advance of $135^{\prime \prime}$ per century above the amount indicated by Newtonian theory. At the time Prof. Newcomb adopted the Hall formula, there appeared to be such an excess of motion of the lunar perigee; but further research, both on the side of theory (Prof. Brown) and on that of observation (Dr. Cowell and others), has emphatically proved that the actual excess is far smaller, and quite consistent with the $2^{\prime \prime}$ per century resulting from Einstein's theory. Thus Brown found $146435^{2} 7^{\prime \prime}$ and $146435^{\prime \prime I}{ }^{\prime \prime}$ for the theoretical centennial motion on two different assumptions of the earth's oblateness (Mon. Notices, vol. Ixiv., p. 532). His discussion gave as the observed value $146435^{2} 3^{\prime \prime}$, while Cowell found $1464353^{\prime \prime}$. In any case, the difference between theory and observation is very much smaller than that required by Hall's law. The latter is thus definitely put out of court, and it becomes a matter of regret that Newcomb adopted it in his tables of the four inner planets. It meant a more drastic alteration of the Newtonian law than that effected by Einstein; the former alters the law in all circumstances, while the latter leaves it unaltered for bodies at rest, but introduces a term that increases the force where there is relative motion.

The second suggestion in explanation of the motion of Mercury's apse is oblateness of the sun. It is easy to show that the attraction of an oblate body falls off more rapidly than the inverse square, thus producing advance of the apse of a satellite. Most of the satellites of the solar system afford examples; the most striking case is Jupiter V., the apse of which makes two entire revolutions in a terrestrial year. Where the satellite does not revolve in the equatorial plane of its primary, there is a second effect of oblateness; it causes the satellite's orbit-plane to shift, its pole describing a circle round the planet's pole. We may refer, for example, to the system of Mars. H. Struve determined the position of the pole of Mars and the amount of oblateness from observations of its satellites; similarly the position of Neptune's pole can be approximately inferred from the change in the orbit-plane of its satellite.

The amount of oblateness of the sun necessary to account for the motion of Mercury's perihelion is not great. Newcomb deduced that the necessary excess of the equatorial diameter over the polar would be slightly more than $\frac{1}{2} / l$; the existence of even this small excess is rendered highly improbable by the very numerous measures of the solar disc, notably the heliometer measures in connection with the Venus-transits of 1874 and 1882; these were discussed by Dr. Auwers, and seemed, if anything, to indicate that the polar diameter is the greater. A further objection is that the solar equator is inclined $3^{\circ} 2 I^{\prime}$ to Mercury's orbit, and its oblateness would produce a diminution of $2 \cdot 6^{\prime \prime}$ per century in the inclination of Mercury's orbit. Observation, if anything, indicates a shift of the inclination in the opposite direction, and the amount $2 \cdot 6^{\prime \prime}$ is so far beyond the probable error as to render the theory of solar oblateness untenable. The above points were established by Newcomb, "Elements of the Four Inner Planets," in 1895 , so that it is strange to find this untenable hypothesis still freely suggested in the United States.

It is fairly obvious that the portion of the zodiacal light that is inside Mercury's orbit would produce effects of the same general kind as those arising from solar oblateness. Now observations of the zodiacal light indicate a smaller inclination to the ecliptic than $7^{\circ}$, the inclination of Mercury's orbit. Thus J. F. J. Schmidt found values ranging from $4^{\circ}$ to $0^{\circ}$, and Prof. Douglas's photograph, taken at Flagstaff, Arizona, on March i9, rgor, shows the light almost symmetrical on each side of the ecliptic. Now, unless the mean plane of the light agreed with that of Mercury's orbit, its gravitational effect on the apse of that planet would be accompanied by a shift of its orbital plane, not verified by observation. It has been found impossible to assign any position to hypothetical perturbing matter that would explain the apse motion of Mercury without causing other anomalies in the elements of that planet and of Venus, which are negatived by observation. Moreover, there is the difficulty that the zodiacal light, if of sufficient mass to produce such an effect, should give us more light than it does. Dr. H. Jeffreys examines the question in Mon. Not., vol. 1xxx., p. $3_{38}$. He shows that if the light arises from reflection by the molecules of a gas, the effect on Mercury would be only I/3000 of that observed. If the light arises from reflection by solid particles, he takes ro $\mathrm{km}$. as their maximum admissible diameter (probably far in excess of what is really tenable); he still finds that their gravitational effect would be only $1 / 20$ of that required. He makes similar calculations for the corona, reaching like conclusions.

Hence we seem to be driven by exhaustion to the Einstein law as the only satisfactory explanation. It clearly can have no effect on orbital planes, so it produces accordance in apsidal motion without introducing other anomalies. Further, it was not an ad hoc hypothesis; it was reached on independent grounds, and it was an undesigned coincidence that it fitted so well for 
Mercury's apsidal motion. For the expression $3\left(\frac{\text { velocity of planet }}{\text { velocity of light }}\right)^{2}$, which is the angular motion of the apse (in fractions of a circumference) per revolution of the planet, involves no empirical or arbitrary constant. We can express the reason for the advance in simple terms thus: At infinity the relative velocity is zero, and the law is the Newtonian one, but the nearer we approach the central orb the higher becomes the velocity, and the greater the extra force. Hence we have another case of the force falling off more rapidly than the inverse square, which we have seen to lead to apsidal advance.

It is interesting to note that the advance per revolution varies as (velocity) ${ }^{2}$ or as $\mathrm{r} / a$. Hence the advance per century varies as $a^{-\frac{5}{2}}$, or it falls off much more rapidly with increase of $a$ than the Hall law, which gives $a^{-\frac{3}{2}}$. In the course of centuries this would discriminate between them, independently of the lunar test; but the orbits of Venus and the earth are so nearly circular that the time for that test has not yet arrived.

In the case of Mars we may note that F. E. Ross's rediscussion of the observations of that planet and of the mass of Venus takes off some $2^{\prime \prime}$ from Newcomb's value of its excess of apsidal motion in a century. When we further remove the Einstein term $1 \cdot 3^{\prime \prime}$, we are left with some $2.7^{\prime \prime}$; as the actually observed quantity is the product of $2 \cdot 7^{\prime \prime}$ by the eccentricity (I/II), it falls well within the limits of observational error.

\section{The Displacement of Solar Lines.}

\section{By Dr. Charlfes E. St. Johr, Mount Wilson Solar Observatory, Pasadena, California.}

TH HE agreement of the observed advance of Mercury's perihelion and of the eclipse results of the British expeditions of 1919 with the deductions from the Einstein law of gravitation gives an increased importance to observations on the displacement of absorption lines in the solar spectrum relative to terrestrial sources, as the evidence on this deduction from the Einstein theory is at present contradictory. Particular interest, moreover, attaches to such observations, inasmuch as the mathematical physicists are not in agreement as to the validity of this deduction, and solar observations must eventually furnish the criterion.

Prof. Eddington's view, if I understand it, is that the theory cannot claim support from the present evidence, nor can observed displacements not agreeing with the theory be on that account regarded as in the slightest degree adverse to it, the only possible conclusion in his view being that there are certain causes of displacement of the lines acting in the solar atmosphere and not yet identified ("Space, Time, and Gravitation," p. I3O).

The great majority of metallic lines observed for differences between their wave-lengths in the sun and terrestrial sources do show displacements. These differ in most cases from those deduced from the Einstein law of gravitation in ways as yet unexplained. If reasonable solar causes can be adduced to account for the wide discrepancies between theory and observation, the position of the generalised theory of relativity would be greatly strengthened.

According to the theory, the displacements are to the red, and are proportional to wave-length, being independent of the intensity of the lines and of the element to which they belong. Observational results differ from those deduced from the theory in at least four important ways. The observed displacements are not proportional to the wave-length; they differ from element to element in the same spectral region; for the same No. 2677 , VOL. IO6] element and spectral region they vary with lineintensity; the displacements show both large positive and negative divergences from the calculated values. Interesting examples are found in Jewell's early work (Astrophysical Journal, vol. iii., p. 89, 1896). The relative values here are of high weight, and the data are important in that the range of elements is wider than occurs in more recent investigations. Divergences in the four directions from the calculated displacements are shown in the following extract from his observations on the differences in wave-length between I 5 solar and arc lines :-

\begin{tabular}{|c|c|c|c|c|c|c|}
\hline & & & Mean $\lambda$ & $\Delta \lambda$ Obs. & $\Delta \lambda$ Cal. & Obs. - Cal \\
\hline Calcium & $\mathrm{H}, \mathrm{K}$, & , 4227 & 4042 & +0.019 & +0.009 & +0.0 \\
\hline Calcium & Int. & $I-6$ & 5227 & +0.004 & +0.011 & -0.007 \\
\hline Iron & , & $10-40$ & $395^{\circ}$ & +o.008 & +0.008 & \pm 0.000 \\
\hline Iron & , & $2-8$ & 3950 & +0.003 & +0.008 & $=0.005$ \\
\hline Aluminium & $"$ & $15-20$ & 3950 & +0006 & +0.008 & -0.002 \\
\hline Nickel & & $10-15$ & 3530 & +0.017 & +0.008 & +0.009 \\
\hline Nickel & 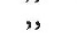 & $1-5$ & 3625 & +0.005 & +0.008 & -0.003 \\
\hline Copper & 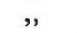 & $6-9$ & 3262 & +0.006 & +0.007 & +0.009 \\
\hline Potassium & , & $00-0$ & 4046 & -0.008 & +0.009 & -0.017 \\
\hline
\end{tabular}

For statistical discussion the quantity of data available is as yet quite inadequate even in the case of iron, the most widely studied element. Not only should the terrestrial and solar wave-lengths be known to high precision over the widest possible range of spectrum, but also the pressure shift per atmosphere. Unfortunately, there are no published data on the wave-lengths and pressure displacements of the iron lines, in which, over a long spectral range, the errors due to pole-effect in the arc are reduced to the magnitude of the calculated Einstein displacement. For other metallic elements the data are even more deficient. With a sufficiently large and varied accumulation of material there is hope that the complex solar conditions may be analysed, and the contributions to the observed effects arising from the various causes determined with some certainty. The pressing need is for data of the requisite accuracy and variety. This need adds interest to determinations of wave-lengths and of pressure dis- 